\title{
Characterization of Poly-Crystalline CdTe Solar Cells Using Aberration-Corrected Transmission Electron Microscope
}

\author{
Tadas Paulauskas ${ }^{1}$, Eric Colegrove ${ }^{1}$, Chris Buurma ${ }^{1}$, Moon $\mathrm{Kim}^{2}$ and Robert F. Klie ${ }^{1}$ \\ 1. University of Illinois at Chicago, Department of Physics, Chicago, USA \\ 2. University of Texas at Dallas, Department of Materials Science and Engineering, Dallas, USA
}

Single CdS/CdTe hetero-junction poly-crystalline photo-voltaic devices are present leaders in thin film solar technology. Commercial success of CdTe-based devices stems from a combination of a nearly ideal direct band gap of the absorber material $(\sim 1.5 \mathrm{eV})$, as well as established manufacturing methods and low cost of the modules, which yield fastest return of investment among currently available technologies. Despite substantial progress in the device engineering and fabrication, the conversion efficiency has seen only minor improvements over the last $\sim 20$ years with the most recent laboratory record held at $\sim 19.6 \%$. Going beyond $20 \%$ efficiency will require breakthrough advances in increasing open circuit voltage, Voc, and minority carrier lifetime. Grain boundaries and intra-grain defects are likely to provide carrier recombination centers but also attract dopants and their influence on a CdTe device performance still needs to be elucidated.

Native defects occurring in typical poly-crystalline thin film CdTe solar cells as well as interfaces in ultra-high-vacuum (UHV) bonded CdTe bi-crystals are studied. Characterization is carried out in the aberration-corrected cold-field emission JEOL JEM-ARM200CF scanning transmission electron microscope (STEM) using high-angle annular dark field (HAADF) and annular bright field (ABF) imaging. The instrument allows $70 \mathrm{pm}$ spatial resolution and $0.35 \mathrm{eV}$ energy resolution at primary electron energy of $200 \mathrm{kV}$. Chemical composition of interfaces and dislocation cores is demonstrated via atomic-column-resolved X-ray energy dispersive spectroscopy (XEDS) using windowless silicon drift detector, the Oxford Instruments X-Max 100TLE.

Figure 1 shows atomic-column-resolved XEDS spectrum image of a coherent twin boundary. Polarity reversal of the Cd-Te dumbbells is clearly observed across the interface. Twins and stacking faults are the most common planar defects in CdTe with very low formation energies $\left(\sim 10 \mathrm{~mJ} / \mathrm{m}^{2}\right)^{1}$. Although twin boundaries do not pose any mid-gap states by themselves ${ }^{1}$, samples without treatment were found be off-stoichiometric across the twin boundary interfaces.

The XEDS map in Figure 2 shows chemical composition of a dislocation core with an unpaired Cd column at the intersection of two faulted $\{111\}$ planes. No Cd-Cd or Te-Te bonds appear across faulted planes or at the core, however the unpaired Cd column is in likely three-fold coordination rather than tetrahedral of the bulk structure. Such cores may possess dangling bonds as was reported for $30^{\circ}$ Shockley partial dislocations ${ }^{2}$.

Figures 3a) and b) show XEDS chemical mapping across a grain boundary in a $\mathrm{CdCl} 2$ treated $\mathrm{CdTe}$ poly-crystalline thin films. Segregation of $\mathrm{Cl}$ towards the grain boundary is evident and it also seem to correlate with a decrease in Te integrated intensity. The presence of $\mathrm{Cl}$ is also detected at dislocation cores as well as at the $\mathrm{CdS} / \mathrm{CdTe}$ interface of $\mathrm{CdCl}_{2}$ treated samples.

[1] Colegrove Eric et al, Journal of Electronic Materials, 41(10), (2012) 
[2] Chen Li et al, Physical Review Letters, 111 096403, (2013)

[3] This research is supported by a grant from the Department of Energy Sunshot Program (DOE DEEE0005956)

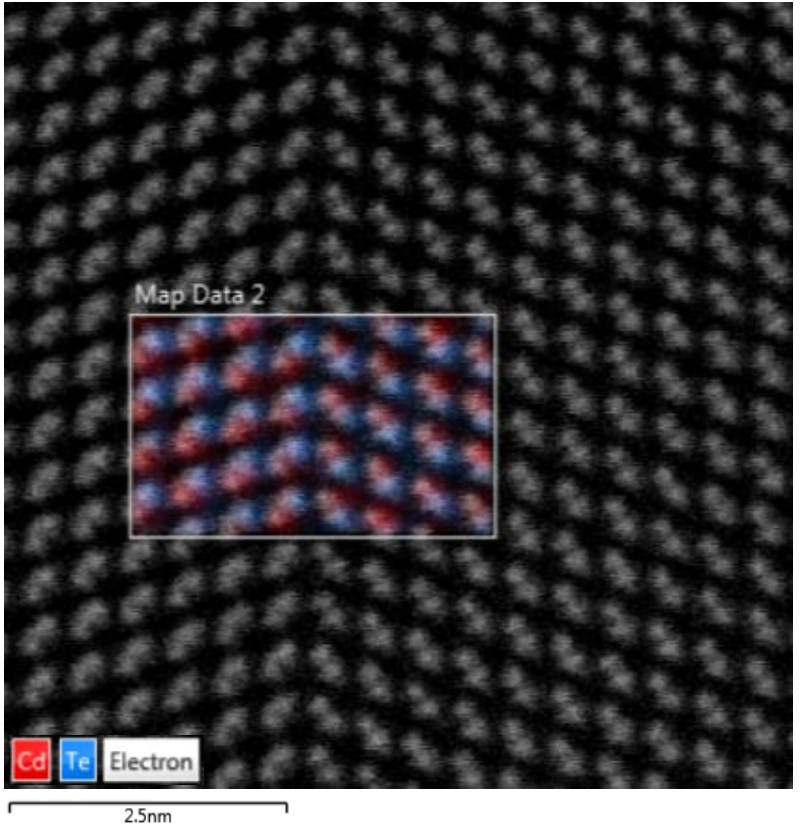

Figure 1. XEDS map of a twin boundary overlaid on HAADF image, $<110>$ zone-axis.
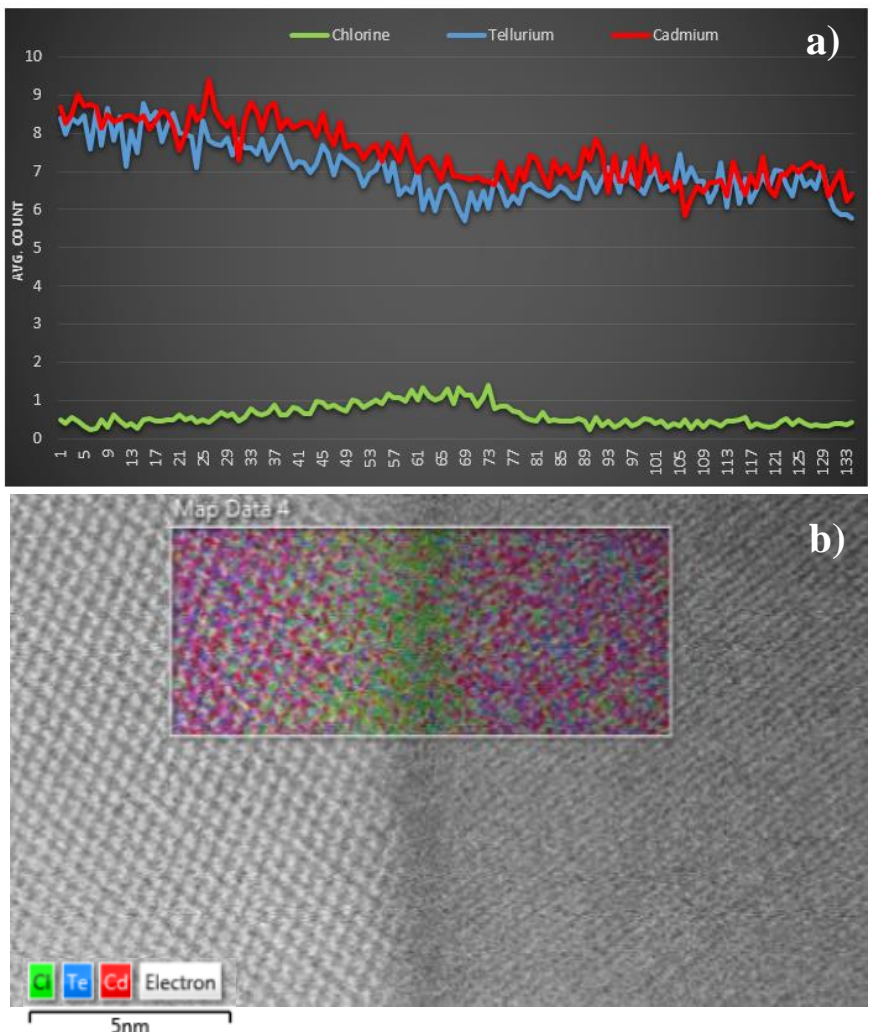

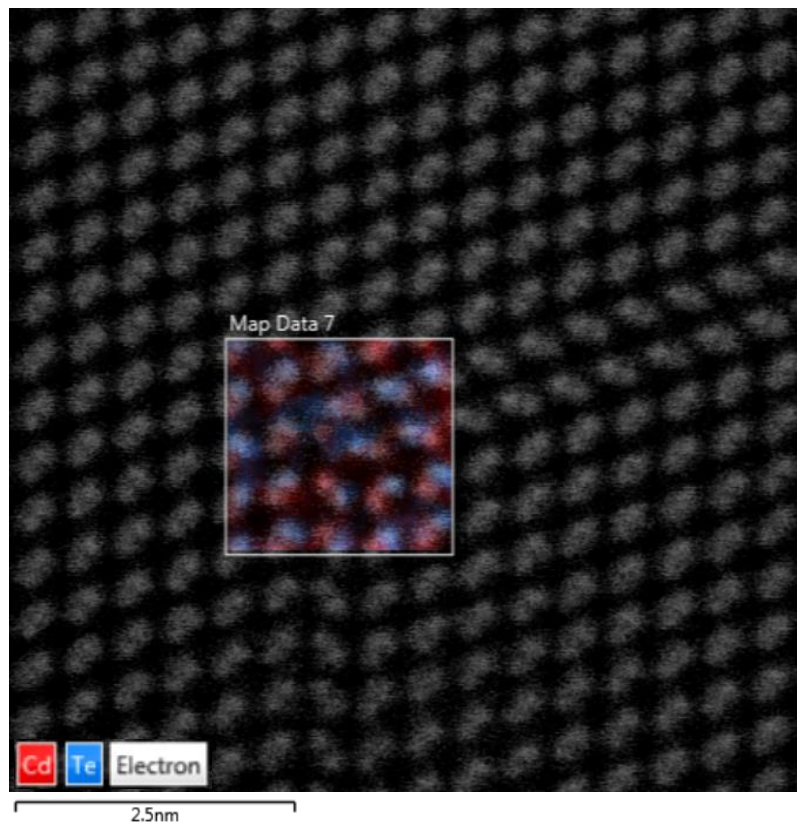

Figure 2. XEDS map of a dislocation core formed by intersection of the faulted planes.

Figure 3.b) Chemical XEDS map showing $\mathrm{Cl}$ segregation along a twist grain boundary in $\mathrm{CdCl} 2$ treated sample. Left grain is viewed along $<110>$.

Figure 3.a) Shows average signal count along the vertical direction vs. position (in pixels). 